\title{
DATA REVIEW OF PATIENTS WITH SPINAL CORD INJURY VISITING OUR OUTPATIENT CLINIC.
}

\author{
Evgenios Diamantidis, Georgios Strougis, Antonios Kontaxakis, Athina-Maria \\ Nella, Helias Karagiannakis, Sofia Fotaki, Dimitra Kagiouli \& Christina-Anastasia Rapidi
}

Physical \& Rehabilitation Department, General Hospital "G.Gennimatas”, Athens, Greece

Aim: Demographic analysis of patients with Traumatic and Non-Traumatic Spinal Cord Injury in the chronic phase.

Material-Methods: Retrospective study of the medical files of patients with $\mathrm{SCl}$ that visited our outpatient clinic in the chronic stage from 2015 to 2016 . Inclusion criteria were patients with $\mathrm{SCl}$ and at least 6 months from time of injury. A total of 134 files were reviewed for further analysis of their demographic data, cause of injury, type of injury, Neurological Level and AIS.

Results: From 134 patients 34\% (46) were female and $66 \%$ (88) were male, from 20 to 81 years old with a mean age of 44 . Time from onset of injury was from 9 months to 50 years with a mean time of 13,6 years from injury(Table 1). Non-Traumatic (NT-SCI) were $36 \%$ (48) and Traumatic (T-SCI) were 64\% (86) (Table 2). Paraplegia was 63\% (85), Tetraplegia was $26 \%$ (35) and $11 \%(14)$ presented with cauda equina syndrome(Table 3$)$. AIS A were $55 \%(74)$ while incomplete lesions (B,C,D) were $45 \%(70)$ in total(Table 3).
Conclusions: Even though contemporary epidemiological studies in SCI seem to favor $\mathrm{NT}^{-S C l}{ }^{1}$ and incomplete lesions ${ }^{2}$, in our day clinic there seems to be a prevalence of $\mathrm{T}-\mathrm{SCl}$ and complete lesions. That could be attributed to the fact that we included patients with lesions in the chronic stage and the fact that patients with high functionality and minor disability stop having regular follow up with a PRM physician.

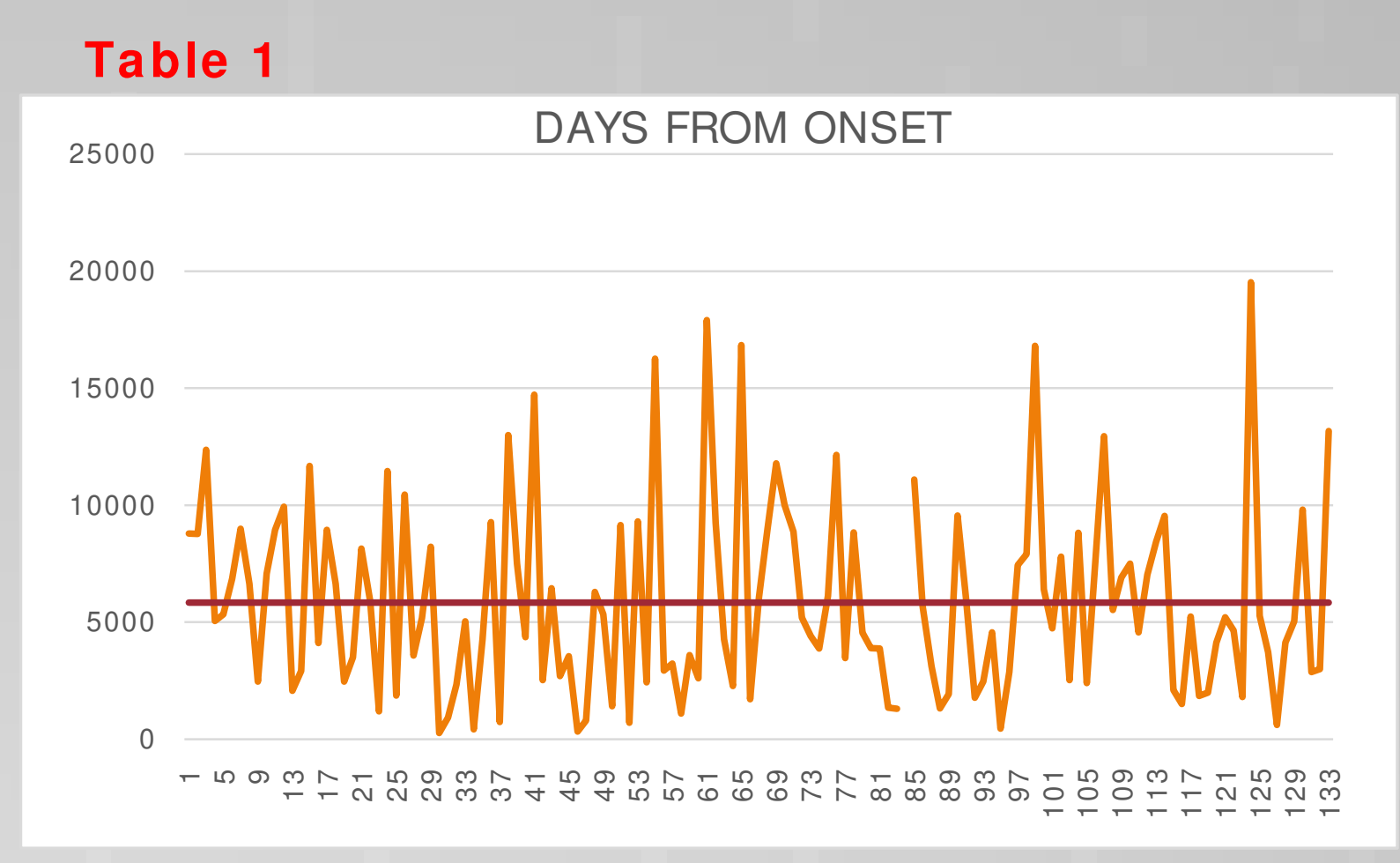

Discussion:

- There is a definite need for a nation wide data base in Greece, in order to have accurate and up to date epidemiologic data concerning SCl

- The above mentioned data can and should be used to create policies, that will reduce the incidence of $\mathrm{SCl}^{3}$
Table 2

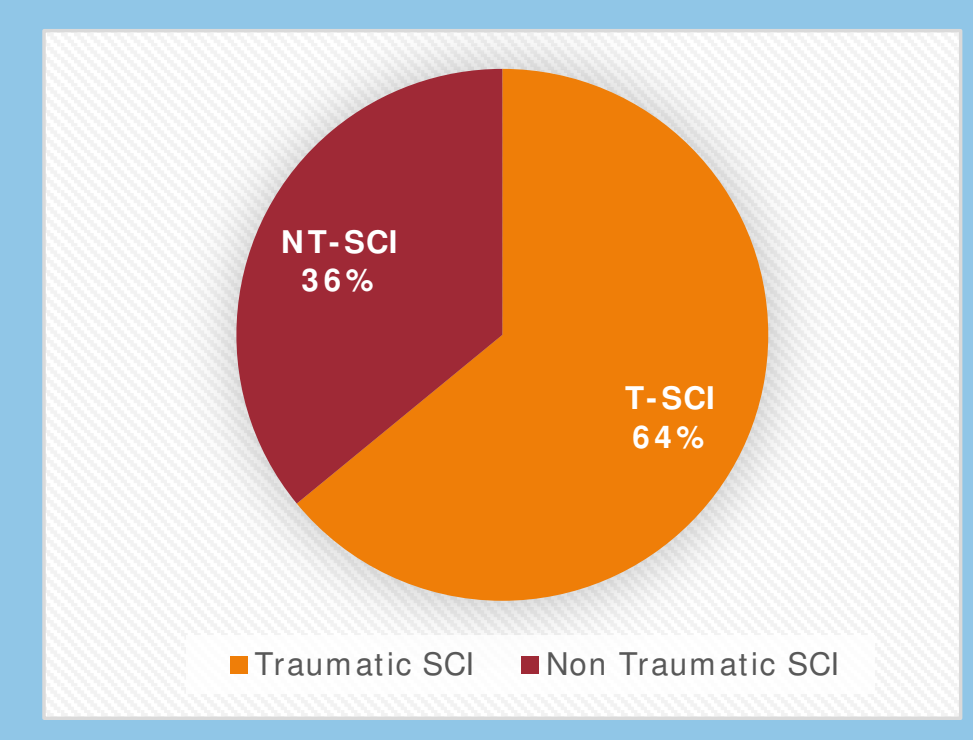

Table 3

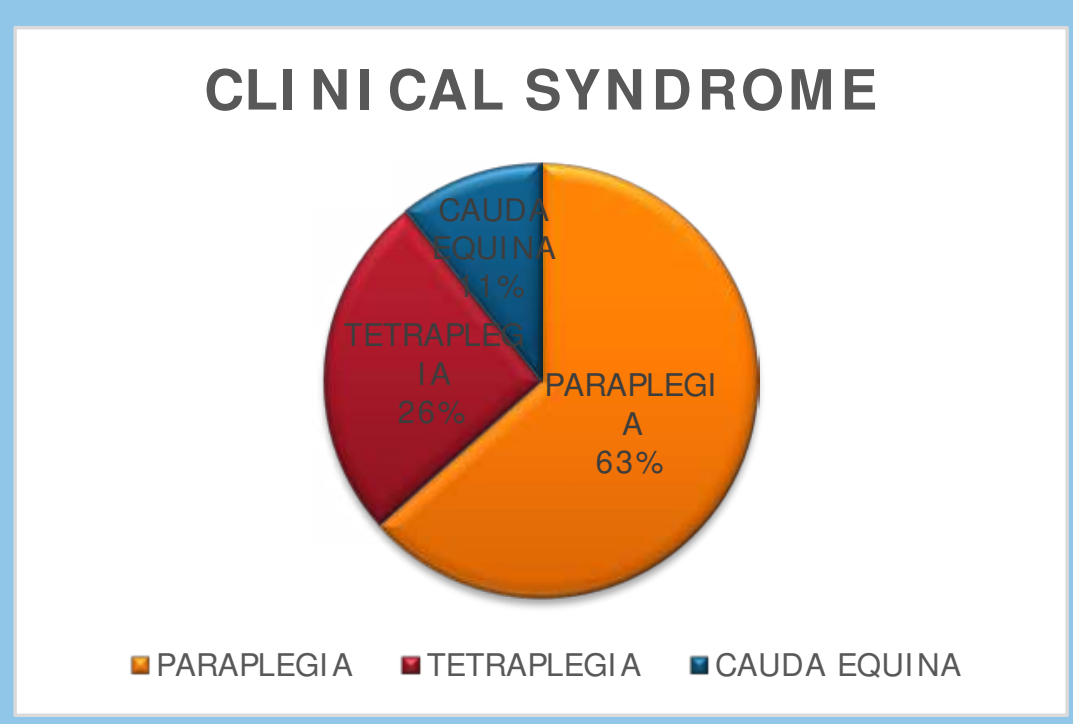

Table 4

AIS CLASSI FI CATI ON

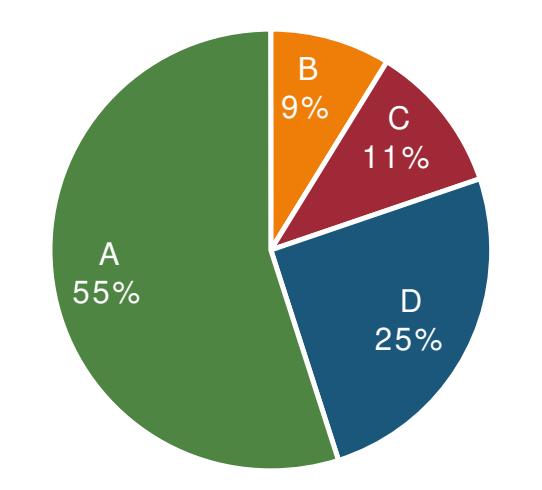

1. Traumatic and Nontraumatic Spinal Cord Injuries- Ge L. et Al. World Neurosurgery 2018 Mar; 111 :e142-e148

2. Epidemiology of traumatic spinal cord injury: trends and future implications- MJ DeVivo. Spinal Cord (2012) 50,365-372

3. Epidemiology of worldwide spinal cord injury: a literature review-Kang Y. et Al. Journal of Neurestoratology Volume 2018:6 Pages 1-9 